\title{
Optimización de los componentes de un sistema de bombeo para comunidades rurales impulsado con energía solar
}

\section{Optimization of the components of a solar powered pumping systemfor rural communities}

\author{
Daniel Querol ${ }^{1}$, Félix Nieto ${ }^{1}$, Rubén Valiente ${ }^{2}$, Reinaldo Erlach ${ }^{3}$ \\ ${ }^{1}$ Programa de Desarrollo Participativo Integral Rural (DEPARTIR), Universidad Nacional Agraria. DQuerol@DEPARTIR.net, \\ ${ }^{2}$ Propietario SERTEVISA, Managua, www.sertevisa.com, ${ }^{3}$ Propietario Agroindustria Taller Electromecánico ATE, Managua, \\ reinharderlach@hotmail.com.
}
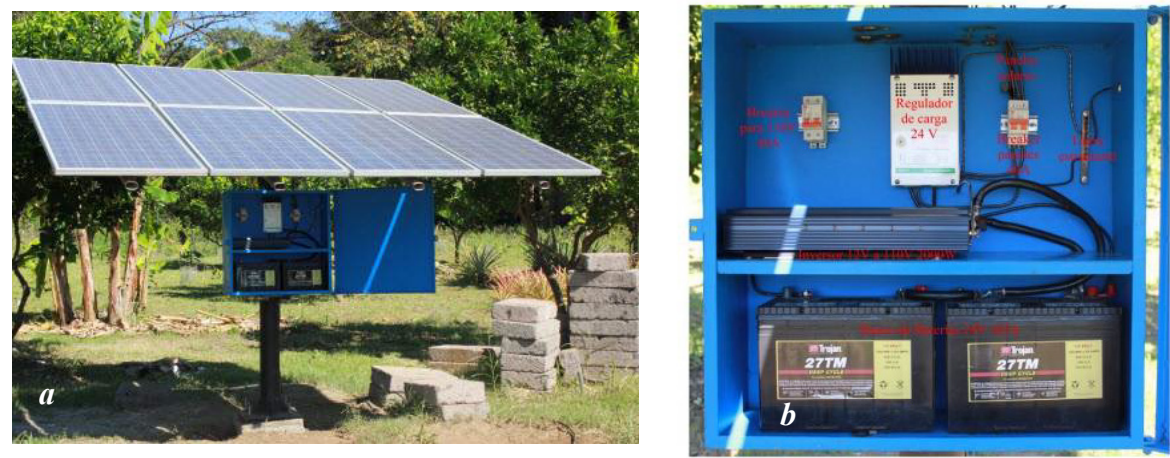

\section{RESUMEN}

Los sistemas de generación de energía eléctrica a partir de paneles fotovoltaicos son una alternativa para zonas rurales aisladas. Se analizaron los elementos y variables que componen un sistema de bombeo impulsado por energía solar en base a las características técnicas y especificaciones de los diferentes componentes y su interacción, para determinar y poder proponer, asociando esta información a características de costos, robustez y disponibilidad, las mejores opciones el bombeo de agua desde un punto de vista de costo/ eficiencia. La solución óptima es la conversión directa a 110 voltios en corriente alterna a partir de la corriente directa almacenada en el mínimo de baterías, con un banco de paneles que corresponda al amperaje a ser consumido por el motor. La bomba periférica tiene un consumo menor de energía por litro bombeado, mientras la altura total de bombeo no rebase los $20 \mathrm{~m}$. Se diseñó y probó un sistema de bombeo abastecido por paneles solares, con un costo de inversión de 2330 US, que bombea entre 6 y $10 \mathrm{~m}^{3}$ de agua al día.

Palabras clave: Bombeo con energía solar, desarrollo participativo, DEPARTIR.

\section{ABSTRACT}

The elements and variables composing a pumping system run with solar energy were analyzed, based on the technical characteristics and specifications of the different components and their interaction, associating this information to cost, robustness and availability, in order to determine and be able to propose the best options for water pumping from a cost/efficiency point of view. The optimal solution is the conversion of DC voltage to $110 \mathrm{~V} \mathrm{AC}$. Panels should generate the energy needed to run the pump, while the battery bank should be the minimum to ensure the amperage needed to start the pump. Peripheral pumps have the lowest energy consumption per pumped liter of water, as long as the total pumping head is below 20 meters. A pumping system run with solar panels was designed and tested, which can pump between 6 and $10 \mathrm{~m} 3$ per day, with an investment of USD 2330.

Key words: Pumping with solar energy, participatory development, DEPARTIR. 
$\mathrm{L}$ os sistemas de generación de energía eléctrica a partir de paneles fotovoltaicos (Paneles Solares) son una alternativa utilizada desde hace varias décadas en las zonas rurales aisladas. Debido a su alto costo de instalación por Watt producido, estos sistemas fueron diseñados para actividades que requerían de un consumo relativamente bajo (iluminación con lámparas fluorescentes y equipos electrónicos).

Durante los últimos años, ha habido una reducción significativa en los costos de los paneles por watt producido, pasando de más de 75 dólares en 1977 a menos de un dólar por watt en 2012 (Carr, 2012). Esto permite explorar nuevos usos de sistemas de generación de energía eléctrica aislados, con mayores consumos. El bombeo de agua para consumo humano es una de

Cuadro 1. Componentes y tipos a ser estudiados

\begin{tabular}{|c|c|c|c|}
\hline Componente & Tipos & Componente & Tipos \\
\hline $\begin{array}{l}\text { 1. Pozo para agua potable } \\
\text { 2. Bomba }\end{array}$ & $\begin{array}{l}\text { Brocal } \\
\text { Mecate } \\
\text { Centrífuga } \\
\text { Periférica } \\
\text { de inyección } \\
\text { sumergible rotativa } \\
\text { sumergible de diafragma } \\
\text { de sumidero }\end{array}$ & $\begin{array}{l}\text { 5. Acumulador } \\
\text { 6. Paneles solares }\end{array}$ & $\begin{array}{l}12 \text { voltios DC } \\
110 \text { voltios AC } \\
220 \text { voltios AC } \\
\text { Onda sinusoidal pura } \\
\text { Onda sinusoidal rectificada } \\
\text { Onda cuadrada }\end{array}$ \\
\hline
\end{tabular}

binaciones posibles. Pare este estudio hemos restringido las opciones a aquellas que nos parecen más razonables, en función de los intereses establecidos en el objetivo del estudio (disponibilidad, calidad relativa, bajo costo inicial, facilidad y bajo costo de mantenimiento y eficiencia).

En una primera aproximación, el sistema de bombeo de agua potable que nos interesa, puede estar compuesto por seis componentes de diferentes tipos (cuadro 1). las principales limitantes

en las comunidades rura-

les, y es generalmente la demanda que viene después de la instalación de una fuente de energía eléctrica.

Existen una serie de programas (homerenergy, 2013; Narejos, 2011) y formas de calcular las características de un sistema de paneles Solares (deltavolt.pe, 2013), en función de las variables locales (latitud, demanda, temperatura, tipo de equipos a ser utilizados, etc.); pero los autores no encontramos un estudio que pudiera ayudar a escoger el sistema de bombeo que minimizara el consumo eléctrico y por lo tanto permitiera la instalación de un sistema de bombeo usando el menor número de paneles fotovoltaicos.

El presente estudio fue impulsado por el Equipo de Tecnologías Amigables (EQUITECNA) del Programa de Desarrollo Participativo Integral Rural (DEPARTIR) de la Universidad Nacional Agraria (UNA), con el apoyo de un ingeniero Electrónico y un ingeniero Electromecánico. El objetivo del presente artículo es analizar los elementos y variables que componen un sistema de bombeo impulsado por energía solar, en base a las características técnicas y especificaciones de los diferentes componentes y su interacción, para determinar y poder proponer, asociando esta información a características de costos, robustez y disponibilidad, las mejores un opciones el bombeo de agua desde un punto de vista de costo/eficiencia.

\section{MATERIALES Y MÉTODOS}

Diseño del estudio. Un sistema de bombeo está compuesto por diferentes tipos de fuentes de agua, bombas, motores o mecanismos que transmiten fuerza a la bomba y fuentes de energía. Esto nos lleva a una gama casi infinita de com-
Decidimos considerar únicamente pozos de brocal, ya que son los más frecuentemente encontrados en las comunidades, aunque los cálculos son igualmente válidos para pozos entubados, siempre y cuando la bomba tenga acceso al agua.

Se calculó de potencia mediante la fórmula:

$\mathrm{P}=\mathrm{V} * \mathrm{I} \quad$ Donde $\quad \mathrm{P}$, es potencia en watts

$\mathrm{V}$, es el voltaje en volts

I, es la corriente en amperes

Las conversiones utilizadas se tomaron de http://www.unitconversion.org/index.html, en especial: Libra por pulgada cuadrada (PSI) corresponde a 0.703 metros de columna de agua (mca), y un galón (gal) es igual a 3.7854 litros.

Las bombas. De los siete tipos de bombas descritos, decidimos no incluir en el estudio algunas bombas debido a los siguientes factores: la bomba de mecate impulsada por motor genera serios problemas de mantenimiento en el sistema de acople; la bomba periférica con sistema de inyección tiene un consumo alto de electricidad y por lo tanto es recomendable acercar la bomba lo más posible al espejo de agua (al igual que en los casos de otras bombas); la bomba sumergible de diafragma detectamos que tenía un consumo muy alto de arranque. Las bombas con motor estudiadas se describen en el cuadro 2. 
REGCORSOSNATURALES

Cuadro 2. Especificaciones de las bombas estudiadas

\begin{tabular}{|c|c|c|c|c|c|c|c|c|c|c|}
\hline Marca & Modelo & Tipo & $\begin{array}{c}\text { Potencia } \\
\text { (hp) }\end{array}$ & $\begin{array}{l}\text { Volumen } \\
\mathrm{g} / \mathrm{m}\end{array}$ & Voltaje & $\begin{array}{l}\text { Succión } \\
\text { pulgadas }\end{array}$ & $\begin{array}{c}\text { Descarga } \\
\text { pulgadas } \\
\text { (A) }\end{array}$ & $\begin{array}{l}\text { Carga } \\
\text { Máxima }\end{array}$ & $\begin{array}{l}\text { Precio } \\
(\$)\end{array}$ & Otros datos \\
\hline Foras & PE50M & Periférica & 0.5 & & 110 & 1 & 1 & 5.5 & 32.15 & $\begin{array}{l}\text { Consumo } \\
370 \mathrm{~W}\end{array}$ \\
\hline Truper & BCAR $3 / 4$ & Periférica & 0.75 & 13.22 & 120 & 1 & 1 & 7.6 & 82.29 & $\begin{array}{l}\text { Altura max. } \\
65 \mathrm{~m}\end{array}$ \\
\hline $\begin{array}{l}\text { Monarch } \\
\text { Pump }\end{array}$ & MJS100 & Centrifuga & 1 & & 115 & 1.25 & 1 & 19.2 & 200.00 & \\
\hline $\begin{array}{l}\text { Pacific } \\
\text { hidrostar }\end{array}$ & 95502 & Sumidero & 1 & 555 & 110 & NNA & 1.25 & & 55.00 & \\
\hline Goulds & 7ЕHO422 & $\begin{array}{l}\text { Pozo } \\
\text { profundo }\end{array}$ & 0.33 & & 220 & NNA & 1 & & 145.00 & $\begin{array}{l}\text { 4.2 A@ } \\
100 \mathrm{psi}\end{array}$ \\
\hline
\end{tabular}

Los motores. No se incluyeron las bombas sumergibles con motor de doce voltios de corriente continua debido a que su costo en el mercado es de 5 a 10 veces mayor que su equivalente con un motor de corriente alterna.
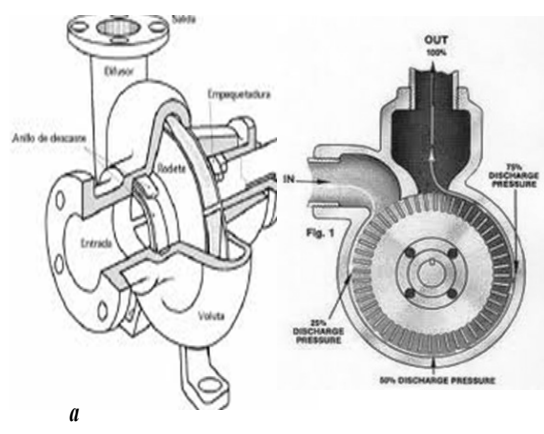

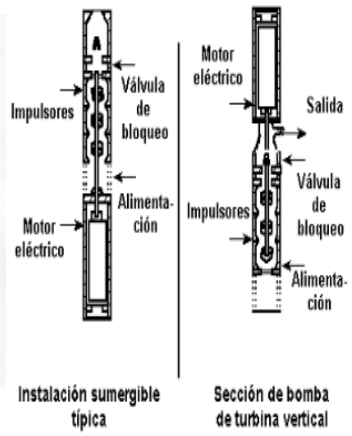

de turbina vertical
Figura 1. Tipos de bombas. a. Bomba centrífuga, b. Bomba periférica, c. Bomba sumergible Olf (2013) y Varios (2014)

Los inversores. Dado que el presente estudio intenta utilizar corriente directa generada por paneles solares y ya que los motores de corriente directa son muy caros en el mercado, decidimos trabajar con una fase intermedia de inversión de corriente que permitiera trabajar con motores de corriente alterna. Los inversores de onda sinusoidal pura son más adecuados para un aprovechamiento óptimo de los motores de corriente alterna, pero su precio actual duplica el costo total de la instalación, Por lo que fueron excluidos del presente estudio.

Los acumuladores y los paneles. En lo referido a los acumuladores y paneles solares, debido a la amplia gama de opciones en el mercado, únicamente comparamos los precios en relación a características técnicas.

Métodos. Para su operación, las características de un sistema de bombeo de agua potable fueron medidas en base a los parámetros descritos en el cuadro 4.

Las bombas y los motores. Las pruebas de bombeo permitieron simultáneamente analizar las características de las bombas y de los motores. Las bombas fueron conectadas en un banco de pruebas utilizando un barril como fuente de agua, un manómetro en línea y una llave para regular las presiones. En la línea eléctrica se instaló un amperímetro y un voltímetro.

Los inversores. Los inversores fueron abiertos para describir sus características internas y posteriormente probadas utilizando una batería de 105Ah con una resistencia (soldador) de 55 watts y un taladro de 1100 watts. En las mediciones de onda se hicieron con un osciloscopio.

Cuadro 3. Caracteristicas de inversores según marca

\begin{tabular}{|c|c|c|c|c|c|c|c|}
\hline Marca & Modelo & Watt & Watt pico & Tipo onda teórico & $\begin{array}{r}\text { Peso } \\
(\mathrm{kg})\end{array}$ & Tipo oscilador & $\begin{array}{l}\text { Protección } \\
\text { fusibles }\end{array}$ \\
\hline NipponAmerica & USADO & 500 & & cuadrada 2 pasos & & KA3525A & $4 * 30 \mathrm{~A}$ \\
\hline Chicago electric & $\begin{array}{l}95596 \\
\text { Inverter }\end{array}$ & 2000 & 4000 & Mod Sine & & Borrado $8 * 40 \mathrm{~A}$ & \\
\hline Xantrex & $\begin{array}{l}1000 \\
\text { placa } \\
\text { DLB44 }\end{array}$ & 1000 & 2000 & Mod Sine & 1.92 & KA3525A & $4 * 30 \mathrm{~A}$ \\
\hline $\begin{array}{l}\text { Power Express } \\
\text { NipponAmerica }\end{array}$ & $\begin{array}{l}\text { (V2.1) } \\
\text { NUEVO }\end{array}$ & $\begin{array}{r}2000 \\
500\end{array}$ & 4000 & $\begin{array}{l}\text { Sinusoidal } \\
\text { cuadrada } 2 \text { pasos }\end{array}$ & & $\begin{array}{l}\text { AS4146A } \\
\text { KA3525A }\end{array}$ & $\begin{array}{l}8 * 25 \mathrm{~A} \\
4 * 30 \mathrm{~A}\end{array}$ \\
\hline
\end{tabular}


Cuadro 4. Componentes y variables seleccionadas

\begin{tabular}{|c|c|c|}
\hline Componentes seleccionados & Variables mecánicas o básicas & Variables eléctricas \\
\hline Pozo para agua potable & $\begin{array}{l}\text { Espejo de agua a } 6 \text { metros de profundidad como máximo** } \\
\text { Capacidad de recuperación infinita** }\end{array}$ & \\
\hline Bomba & $\begin{array}{l}\text { Altura de bombeo } 0 \text { a } 70 \mathrm{~m}(0 \text { a } 100 \mathrm{psi})^{* *} \\
\text { Volumen bombeado por minuto* } \\
\text { Altura máxima de bombeo* }\end{array}$ & \\
\hline Motor eléctrico & $\begin{array}{l}\text { Caballaje reportado* } \\
\text { Caballaje producido* }\end{array}$ & $\begin{array}{l}\text { Consumo al arranque* } \\
\text { Consumo con carga* }\end{array}$ \\
\hline Inversor & Calidad diseño* & $\begin{array}{l}\text { Consumo* } \\
\text { Calidad diseño* } \\
\text { Protección* } \\
\text { Calidad componentes* } \\
\text { Robustez* }\end{array}$ \\
\hline Acumulador & & Cuantos* \\
\hline Paneles solares & & $\begin{array}{l}\text { Cuantos* } \\
\text { Interacción entre inversores }\end{array}$ \\
\hline y motores* & & \\
\hline
\end{tabular}

\section{RESULTADOS Y DISCUSIÓN}

Se presentan a continuación los resultados del estudio por componentes. En el caso de las bombas, por razones prácticas tuvimos que analizarlas junto con los motores.

Las bombas y los motores. Uno de los supuestos básicos para poder realizar este trabajo es que las diferencias entre los motores y las bombas no están determinadas por las marcas, ya que por experiencia de uno de los autores, las diferencias entre marcas para el mismo tipo de bomba con el mismo caballaje son menores al 5\%. Por lo tanto solo se comparan los tipos de bombas y su caballaje.
Como se puede ver en los resultados presentados en el cuadro 5, cada una de las bombas tiene características que deben ser tomadas en cuenta según los objetivos del proceso de bombeo. Los factores determinantes serán la altura de bombeo necesaria y el volumen esperado. Queda claro por ejemplo que las bombas de sumidero solamente podrán funcionar para alturas menores de cinco metros, mientras que será necesario utilizar bomba de pozo profundo para llevar agua más de 40 metros, altura máxima de bombeo de la bomba centrífuga. 
REGCORSOSNATURALES

Cuadro 5. Medición de presiones, consumo y débito

\begin{tabular}{|c|c|c|c|c|c|c|}
\hline & Tipo de Bomba & $\begin{array}{c}\text { Periférica } \\
1 / 2 \mathrm{hp}\end{array}$ & $\begin{array}{c}\text { Periférica } \\
3 / 4 \mathrm{hp}\end{array}$ & $\begin{array}{c}\text { Centrifuga } \\
1 \mathrm{hp}\end{array}$ & $\begin{array}{l}\text { Sumidero } \\
1 \mathrm{hp}\end{array}$ & $\begin{array}{l}\text { Pozo profundo } \\
1 / 3 \mathrm{hp}\end{array}$ \\
\hline & hp & 0.5 & 0.75 & 1 & 1 & 0.33 \\
\hline \multirow[t]{4}{*}{ OPSI } & Amp Arranque & 5.33 & 13.5 & 38 & 18 & 7.5 \\
\hline & Amp Trabajo & 4 & 3 & 14 & 5 & 3 \\
\hline & Voltaje & 102 & 103 & 103 & 103 & 210 \\
\hline & Caudal 1/min & 45 & 39 & 103 & 62 & 51 \\
\hline \multirow[t]{4}{*}{ 10PSI } & Amp Arranque & 5 & 14 & 34 & & \\
\hline & Amp Trabajo & 4 & 3 & 15 & & \\
\hline & Voltaje & 103 & 103 & 99 & & \\
\hline & Caudal 1/min & 25 & 34 & 87 & & \\
\hline \multirow[t]{4}{*}{ 15PSI } & Amp Arranque & 5 & 14 & 39 & & \\
\hline & Amp Trabajo & 3 & 3.5 & 17 & & \\
\hline & Voltaje & 103 & 103 & 93 & & \\
\hline & Caudal 1/min & 8.5 & 30 & 87 & & \\
\hline \multirow[t]{4}{*}{ 20PSI } & Amp Arranque & & 14 & 36 & & 7.5 \\
\hline & Amp Trabajo & & 3.5 & 17 & & 3 \\
\hline & Voltaje & & 103 & 97 & & 210 \\
\hline & Caudal 1/min & & 24 & 86 & & 49 \\
\hline \multirow[t]{4}{*}{ 30PSI } & Amp Arranque & & 14 & 35 & & 8 \\
\hline & Amp Trabajo & & 3.5 & 18 & & 3 \\
\hline & Voltaje & & 103 & 99 & & 210 \\
\hline & Caudal 1/min & & 13 & 80 & & 47 \\
\hline \multirow[t]{4}{*}{ 40PSI } & Amp Arranque & & & 36 & & 8 \\
\hline & Amp Trabajo & & & 18 & & 3 \\
\hline & Voltaje & & & 95 & & 210 \\
\hline & Caudal 1/min & & & 59 & & 42 \\
\hline \multirow[t]{4}{*}{ 60PSI } & Amp Arranque & & & 30 & & 8 \\
\hline & Amp Trabajo & & & 16 & & 3 \\
\hline & Voltaje & & & 98 & & 210 \\
\hline & Caudal 1/min & & & 11 & & 34 \\
\hline \multirow[t]{4}{*}{ 80PSI } & Amp Arranque & & & & & 8 \\
\hline & Amp Trabajo & & & & & 3 \\
\hline & Voltaje & & & & & 210 \\
\hline & Caudal 1/min & & & & & 25 \\
\hline \multirow[t]{5}{*}{ 100PSI } & Amp Arranque & & & & & 7.5 \\
\hline & Amp Trabajo & & & & & 2.3 \\
\hline & Voltaje & & & & & 210 \\
\hline & Caudal 1/min & & & & & 7.9 \\
\hline & $\begin{array}{l}\text { Presión PSI } \\
\text { caudal } 0 \text { lpm }\end{array}$ & & 18 & 40 & 65 & 105 \\
\hline
\end{tabular}

Las bombas periféricas, de muy bajo costo, pueden bombear adecuadamente hasta una altura de 11 o 21 metros, para un caballaje de $1 / 2$ y $3 / 4$ de hp respectivamente. La bomba de sumidero de $1 \mathrm{hp}$ bombea 45 litros por minuto sin presión, pierde eficiencia antes de poder elevar agua a mas de 6 metros y por lo tanto no debe ser considerada como una alter- nativa. La bomba centrífuga de un caballo bombea más del doble que cualquiera de las bombas periféricas mientras que la bomba del pozo profundo no se asegura puede bombear desde profundidades mayores. El significado del cuadro $5 \mathrm{se}$ puede ver claramente en la figura 1. 


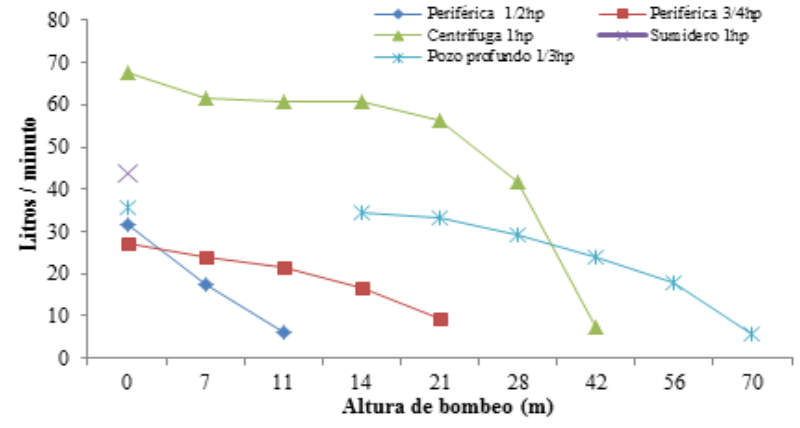

Figura 1. Capacidad de bombeo (litros por minuto en función de altura de bombeo) por tipo de bomba.

Sin embargo la figura 1 , que nos da una información sobre la capacidad de las bombas, no toma en cuenta el factor que será determinante para seleccionar una bomba en un sistema con generación fotovoltáica, a saber, la eficacia relativa de bombeo en función de consumo de electricidad. Para ello graficamos (Figura 2) los litros bombeados por minuto por cada kilowatt consumido, en función de la altura de bombeo.

La figura 2 es más interesante para determinar el tipo de bomba a seleccionarse en función de la altura y volumen de bombeo necesarios, asegurando el mínimo consumo de electricidad. El uso de bombas del tipo periférico es óptimo mientras las alturas de bombeo no sean superiores a los quince metros. La bomba centrífuga es la menos eficiente y la bomba del pozo profundo debe ser utilizada cuando se bombea agua desde profundidades de más de 10 metros. Debemos recordar que, a excepción de las bombas sumergibles, todas las bombas empiezan a generar problemas cuando succionan más de seis o siete metros, por lo que en el caso de las bombas periféricas y centrífugas, se deberá instalar la plataforma del motor a menos de seis metros del espejo de agua.

Los inversores. Durante la observación de los inversores con el osciloscopio (cuadro 6), se detectaron diferencias en cuanto a la forma de la onda, la frecuencia varió relativamente poco y dos de los inversores tuvieron un pequeño consumo aun sin estar conectados a equipo alguno.

Detectamos un problema de diseño en algunos de los inversores revisados ya que en el afán de ahorro se realizan circuitos que no producen una onda sinusoidal aproximada sino que en la práctica parece ser una onda cuadrada, con la consiguiente pérdida de efectividad y alejándose de los valores teóricos rápidamente, lo cual para una aplicación sin un consumo excesivo puede ser que pase inadvertida, pero a la hora de exigírsele según sus especificaciones los defectos afloran rápidamente.

Adicionalmente la calidad de los componentes es un factor adicional que juega en contra del buen desempeño de estos equipos, siendo este un problema generalizado en la industria electrónica producto de los componentes de mala calidad fabricados a gran escala.

Con carga, el equipo con onda sinusoidal fue significativamente más eficiente, consumiendo entre 20 y $80 \%$ menos que los equipos con onda cuadrada u onda cuadrada de dos pasos.

Al valorar la calidad, robustez y diseño electrónico y comparar el ranking como el precio en dólares por watts, podemos concluir que, para los equipos estudiados, hay una correlación directa entre el precio por watt y la calidad del inversor.

Cuadro 6. Características medidas y valoración de inversores

\begin{tabular}{|c|c|c|c|c|c|c|}
\hline & & $\begin{array}{l}\text { Nippon } \\
\text { América }\end{array}$ & $\begin{array}{l}\text { Chicago } \\
\text { electric }\end{array}$ & Xantrex & $\begin{array}{l}\text { Power } \\
\text { Express }\end{array}$ & $\begin{array}{l}\text { Nippon } \\
\text { America }\end{array}$ \\
\hline \multirow[t]{5}{*}{ Osciloscopio } & Imagen de onda & $\begin{array}{l}\text { cuadrada } \\
\text { con picos } \\
\text { irregular }\end{array}$ & $\begin{array}{l}\text { cuadrada } 2 \\
\text { pasos }\end{array}$ & Cuadrada & $\begin{array}{l}\text { Casi } \\
\text { sinusoidal }\end{array}$ & $\begin{array}{l}\text { cuadrada } \\
\text { con picos } \\
\text { irregular }\end{array}$ \\
\hline & Volt vacío & 70 & 108 & 125 & 123 & 132 \\
\hline & Frecuencia Vacío & 80 & 60 & 69 & 60 & 60 \\
\hline & Amp vacío & 0 & 0.1 & 0 & 0 & 0.1 \\
\hline & Volt RMS* & 58 & 75 & 82 & $40-73$ & \\
\hline \multirow[t]{2}{*}{ Consumo } & Consumo con $0.5 \mathrm{~A}(\mathrm{~A})$ & 9 & 0.9 & 0.4 & 0.2 & \\
\hline & Consumo con $10 \mathrm{amp}(\mathrm{A})$ & NA & 12.8 & 10 & 9 & NA \\
\hline \multirow[t]{6}{*}{ Valoración } & Calidad comp. /10 & 5 & 6 & 5 & 5 & 5 \\
\hline & Robustez / 10 & 5 & 7 & 8 & 7 & 5 \\
\hline & Diseño electronico / 10 & 3 & 5 & 8 & 8 & 3 \\
\hline & Ranking & 5 & 3 & 2 & 1 & 4 \\
\hline & Precio en internet & 90 & 209 & 100 & 155 & 90 \\
\hline & US/Watt & 5.56 & 9.57 & 10.00 & 12.90 & 5.56 \\
\hline
\end{tabular}

* RMS es la forma de medir la potencia efectiva en circuitos de corriente alterna, quiere decir "raíz cuadrada media" y es un procedimiento matemático por medio del cual nos hacemos a la idea de que tanta potencia tenemos a nuestra disposición en un equipo dado. Sin embargo existen muchos fabricantes y mercadotécnicos que suelen usar la medida denominada PMPO o comúnmente conocida como "pico a de milisegundos o menos, lo cual quiere decir que es una med que puede tener la sinusoide por periodos potencia real de un equipo y tiende a engañar a los consumidores quienes se dejan llevar por los grandes números con que ofertan sus equipos sin fijarse si son watts RMS o PMPO.

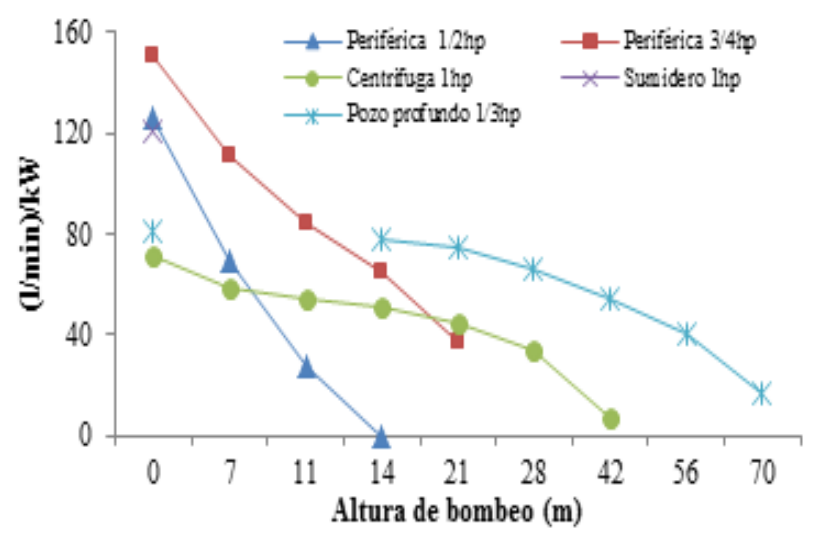

Figura 2 Litros bombeados por minuto por kilowatt consumido según altura de bombeo. 
Los acumuladores y los paneles. Los acumuladores (baterías) sirven en este diseño esencialmente para proteger al inversor de voltajes excesivos o por debajo de los necesarios viniendo de los paneles.

Dado que el bombeo de agua no necesita hacerse de noche, la cantidad de acumuladores estará determinada por el tiempo autonomía adicional necesaria y como un mecanismo de almacenamiento de energía generada que no pueda ser aprovechada por la bomba de manera inmediata.

Los paneles solares hoy en día tienen un costo directamente proporcional a los watts producidos, siendo la única variante el precio del tipo de celdas utilizadas, la cual determina la eficiencia por metro cuadrado (Solar Choice, 2009). La eficiencia es medida como porcentaje de conversión de la energía solar recibida por el panel y convertida en electricidad. Los tres principales tipos de celdas solares tienen la siguiente eficiencia: Monocristalinos, 15-20\%, 135-170 Watts $/ \mathrm{m}^{2}$; policristalinos, $13-16 \%, 120-150$ Watts $/ \mathrm{m}^{2}$ y celdas de película delgada, 7-13\%, 60-80 Watts $/ \mathrm{m}^{2}$.

En nuestro caso no necesitamos comprar los paneles de alto rendimiento por unidad de superficie ya que no tenemos limitantes de espacio. El factor determinante es el precio por watt generado, que en septiembre del 2013 estaba en un mínimo de 0.58 US por watt en Miami (ecobusinesslinks, 2013).

Propuesta de sistema y cálculo de costos. Un sistema económico y de fácil mantenimiento estaría compuesto por una bomba periférica, un inversor con al menos cinco veces la capacidad en watt del consumo normal de la bomba, un acumulador con suficiente capacidad para la operación de la bomba durante media hora y paneles solares suficientes para mantener la bomba operando durante el tiempo necesario de bombeo diario. Este sistema ha sido establecido con buen suceso en la Universidad Nacional Agraria.

Un sistema de estas características bombea 1080 litros de agua por hora, con una elevación total de 8 metros, permitiendo por lo tanto el bombeo de entre 6 y 10 metros cúbicos (1600 a 2600 galones, 29 a 48 barriles) al día con buena insolación. Esto permitiría regar por goteo entre media y una hectárea de tomate (6000 a 10000 plantas).

Debido al consumo de la bomba, realmente solo se necesitan la mitad de los paneles para hacerla funcionar, quedando el resto de la capacidad instalada para otros usos. Con cuatro paneles el costo total del sistema sería de USD 2 130.00 (cuadro 7)

\section{CONCLUSIONES}

Debido al número de variables y a las múltiples opciones en el mercado, iniciamos con un gran número de posibilidades de diseño del sistema.

Las bombas periféricas fueron eficientes bombeando hasta alturas de 20 metros. Si la altura de bombeo es mayor, se deberá optar por una bomba del pozo profundo. El caballaje estará determinado por la cantidad de agua que se quiera bombear por minuto.

Un factor importante a tomar en cuenta es el amperaje demandado por el motor durante el arranque, que en el caso de un inversor de onda cuadrada será multiplicado muy por encima de la capacidad normal de arranque del inversor.

Un sistema económico y de fácil mantenimiento estaría compuesto por una bomba periférica, un inversor con al menos cinco veces la capacidad en watt del consumo normal de la bomba, un acumulador con suficiente capacidad para la operación de la bomba durante media hora y paneles solares suficientes para mantener la bomba operando durante el tiempo necesario de bombeo diario.

La capacidad de bombeo del sistema es de 1080 litros de agua por hora, con una elevación total de 8 metros, con bombeo de entre 6 y 10 metros cúbicos (1600 a 2600 galones, 29 a 48 barriles) al día con buena insolación.

El sistema permitiría regar por goteo entre media y una hectárea de tomate (6000 a 10000 plantas).

Debido al consumo de la bomba, se necesitan la mitad de los paneles para hacerla funcionar, quedando el resto de la capacidad instalada para otros usos. 


\section{REFERENCIAS BIBLIOGRÁFICAS}

Carr, G. 2012. The economist. (en línea). consultado 21 nov. 2012. Disponible en http://www.economist.com/news/21566414-alternative-energy-will-no-longer-be-alternative-sunny-uplands.

Deltavolt.pe. 2013. Calculadora para dimensionar sistemas fotovoltaicos. (en línea). Disponible en http://deltavolt.pe/calcsolar Ecobusinesslinks. 2013. Free-solar-panel-price-survey. (en línea). consultado 09 sep. 2013. Disponible en http://www.ecobusinesslinks.com/surveys/free-solar-panel-price-survey/.

Homerenergy. 2013. www.homerenergy.com. [En línea] 2013. http://homerenergy.com/documentation.html.

Narejos, A. 2011. Programas-gratuitos-para-calcular-instalaciones-fotovoltaicas. (En línea). Consultado 6 jun. 2011. Disponible en http://antonionarejos.wordpress.com/2011/06/06/programas-gratuitos-para-calcular-instalaciones-fotovoltaicas/.

Olf, J. 2013. Pumpfundamentals. (en línea). Consultado 9 sep. 2013. Disponible en http://www.pumpfundamentals.com/pump_ glossary.htm

Solar Choice. 2009. Which solar panel type best suits your needs - monocrystalline, polycrystalline or amorphous thin film? (en línea). Consultado 29 ene. 2009. Disponible en http:/www.solarchoice.net.au/blog/which-solar-panel-type-best-suits-your-needs-monocrystalline-polycrystalline-or-amorphous-thin-film/.

Varios. (2014). Guía para el desarrollo de proyectos de bombeo de agua con energía fotovoltáica. Obtenido de http://solar.nmsu. edu/wp_guide/bombeo.html 